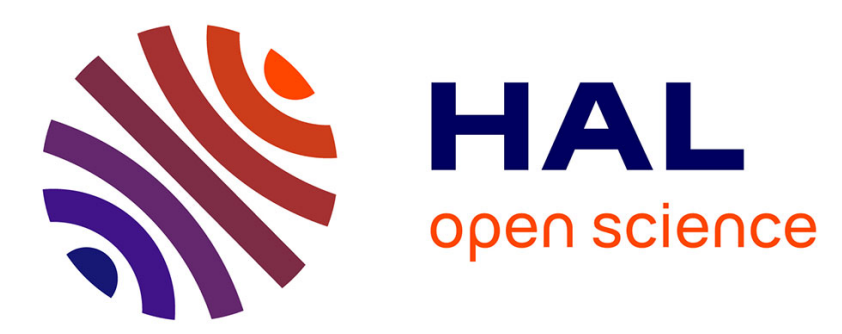

\title{
Homotopy switching model for dyad haptic interaction in physical collaborative tasks
}

\author{
Paul Evrard, Abderrahmane Kheddar
}

\section{To cite this version:}

Paul Evrard, Abderrahmane Kheddar. Homotopy switching model for dyad haptic interaction in physical collaborative tasks. WHC'09: World Haptics - 3rd Joint EuroHaptics conference and Symposium on Haptic Interfaces for Virtual Environment and Teleoperator Systems, Mar 2009, Salt Lake City, Utah, United States. pp.45-50, 10.1109/WHC.2009.4810879 . lirmm-00796731

\section{HAL Id: lirmm-00796731 https://hal-lirmm.ccsd.cnrs.fr/lirmm-00796731}

Submitted on 4 Mar 2013

HAL is a multi-disciplinary open access archive for the deposit and dissemination of scientific research documents, whether they are published or not. The documents may come from teaching and research institutions in France or abroad, or from public or private research centers.
L'archive ouverte pluridisciplinaire HAL, est destinée au dépôt et à la diffusion de documents scientifiques de niveau recherche, publiés ou non, émanant des établissements d'enseignement et de recherche français ou étrangers, des laboratoires publics ou privés. 


\section{Homotopy Switching Model for Dyad Haptic Interaction in Physical Collaborative Tasks}

\author{
Paul Evrard \\ CNRS-LIRMM \\ AIST/CNRS JRL
}

\author{
Abderrahmane Kheddar \\ CNRS-LIRMM \\ AIST/CNRS JRL
}

\begin{abstract}
The main result of this paper is a new model based on homotopy switching between intrinsically distinct controllers, to encompass most behaviors encountered in dyadic haptic collaborative tasks through an intermediate object. The basic idea is to switch continuously between two distinct extreme behaviors (leader and follower) for each individual, which creates an implicit bilateral coupling within the dyad. The physical collaborative interaction is then described only with two distinct homotopy time-functions that vary independently. These functions can likely describe the signature of a collaborative task. A virtual reality haptic set-up is used to assess the proposed theory.
\end{abstract}

Index Terms: K.6.1 [Haptic interaction]: Collaborative physical tasks-Modeling; K.7.m [Miscellaneous]: Ethics-

\section{INTRODUCTION}

One of the characteristics of humans is their ability to collaborate to perform tasks. Collaborative tasks range from searching an object, piloting a plane to object passing, dancing, assembly tasks or daily bulky or heavy object transportation, etc. In this work, we are interested by tasks which involve physical contact between partners, typically manipulation tasks where more than one agent act on an object of interest to the task. More specifically, our main target is to achieve a similar task on virtual or robotic avatars such as humanoids (graphical or robotics ones).

If it is to achieve physical collaboration between two programmable avatar agents (i.e. dyad of robots or virtual figures), a pure engineering approach would certainly outcome with several possible models and control algorithm strategies, that may or may not rely on a well defined communication protocol between the members of these dyads. Automating physical collaboration between robots and virtual avatars is not that hard compared to the similar problem in which one actor of the dyad is a human whereas the collaborator is a virtual or a robotic avatar. This problem is a typical case study where a cognitive approach is needed. By cognition we do not necessarily mean to adapt models inspired from dyadic physical interaction (providing existence of such models), but rather a deep understanding of the sensory-motor and communication mechanisms and strategies we humans use to perform physical interaction. This is indeed very helpful in order, for any programmable avatar, to: (i) endow capabilities of identification, interpretation and eventually prediction of its human partner's intentions and actions, and (ii) communicate its own programmed intentions.

In robotics, several works addressed human-robot partnership in physical interaction. It has several names, but more recurrent ones translate collaborative manipulation. Many control schemes have been implemented for robots as partners or helpers. Most of the controllers are based on impedance models, see [6][8]. These control schemes can be improved by varying the impedance parameters [18][3], by adding constraints on the system so that its dynamic behavior is familiar to the human operator [1][5], or by making the robot actively assist the human operator [9][2].

A recurrent assumption of all existing approaches is to appoint $a$ priori a unilateral distribution of the roles to the partners that will not change all along the task execution. In most general cases, the leader's role is assigned to human operator (or to one robot when no human is involved in the task), and a follower's role to all other agents. Some researchers [12], however, suggest that the contribution to the motion of the manipulated object can be distributed between partners, but studied the case where a robot follower collaborates with a human leader. Although more or less advanced control schemes have been proposed where the robot can actively assist the human operator [9], and vary its level of assistance [2], an input is still needed from the human operator as the motion of the robot will be based on the intentions of the humans which always leads the task.

Studies dealing with human-human physical interaction are surprisingly few compared to human motion in a standalone role. An excellent recent work made by Reed et al. [16] summarizes in a nearly exhaustive way the state-of-the-art related to this issue. Moreover, they made a considerable contribution to see clearer inside this problem. Among their notable contributions, the observed specialization in dyads performing a physical common task is of interest. They observed that dyads specialize in sub-tasks probably to reduce task complexity or resolve redundancy. However, this interesting observation raises several unanswered questions: on what basis does specialization occur? For a given task, who will specialize on what and why? Specialization also occurs after several trials, and might also outcome from learning or optimization processes. This, plus the lack of a clear model might explain why the porting of the specialization process on an automated machine was not trivial and totally successful [13]. Among other interesting issues of Reed's work, we noted the link implicitly established between internal forces as a hypothetical haptic cue for communication. This idea also links the way impedance has been adjusted in [3]. We make use of this knowledge in our approach.

Relative to previous work which certainly inspired several aspects our own model, we focus on manipulation tasks which involve a human partner and either a virtual or a robotic partner to realize complex physical tasks. We have in mind two main applications: cognitive interactive games implying virtual figures (including virtual humanoids) and collaborative physical tasks implying a human and a robotic humanoid either in a standalone or in a telepresence mode [11]. We name haptic collaboration scenarios between a dyad of humans by the acronym person-object-person (POP). In this work however we propose and examine an open-loop model which will encompass most possible behaviors encountered in POP situations that easily translate to a person-object-avatar (POA) programmable model. The driving ideas behind our proposed POP/A model are as follows:

- a totally symmetric model to translate interchangeability of the roles within dyads in POP;

- a template model that encompasses most encountered behaviors in physical interaction (conflicts, passive or full proac- 
tive actors, specialization as proposed by Reed et al. [16], and others;

- independent from a dyad's internal control models: this issue is important to ensure portability and non-subjectivity of the proposed model from POP to POA;

- simple and tunable at will.

The remainder of the paper presents our model through insights and a mathematic basis; then we provide examples of implementation on a virtual avatar performing a collaborative task with a human. We chose a priori parameters to exemplify its use. Note that like many researches our model also raises several questions that need further investigations, we discuss some of them in the conclusion.

\section{A HOMOTOPY SWITCHING MODEL FOR POP}

In this section, we examine a new model that encompasses most behavioral situations that are encountered in POP. First, we will provide insights for understanding the idea which is behind the model, and then we provide mathematical foundations on which our theory is built. Finally we present a template abstract model for POP with some examples.

\subsection{Insights}

It is generally assumed that in performing a physical collaborative task, each operator can behave independently as a leader or a follower. These are extreme cases that have been extensively used in robotic to implement controllers of human-object-robot collaborative tasks. It is certainly easier to affix a robot to behave in either one of these cases. This is made simply by programming the appropriate controller. However, it is nearly impossible to force the human to behave in an exclusively passive role. Therefore, in robotics, this problem has recurrently been solved by programming robots to be passive and assigning the human operator the leadership in the task.

There are plenty of simple situations, which can be guessed easily by the reader, where this is not possible. For example, consider a given task function space with a set of constraints. In most common situations, these constraints (even when they hold and do not vary all over the task execution time) would certainly induce a given operator to be leading in a sub-set of a given task space and following in some others. Then, the other operator will ideally behave in a complementary way, that is, s/he is also both leader and follower at the same time: therefore, in POP, both operators can be leading and following at the same time.

Another point to highlight is that the distribution of the leadership for the different subtasks does not need to be affixed. Depending on the context, on the amount of information available, each partner may choose to give up or claim for the leadership of a subtask. This can especially occur if one partner is close to violate one of his own constraints that are not directly related to the collaborative task. For example, a robot which follows a human may come close to a joint limit or singularity which will prevent it from behaving as the human intends to. Before it happens and the robot loses manipulability, it may claim for leadership and handle the subtask in a way that will keep it far from its limits (of course this requires that the task can be achieved in several ways).

This role switching should occur in a smooth way, so that i) the human partner has time to react and to negotiate progressively the role sharing and ii) the motion of the robot is not abrupt and jerky. Moreover, a smooth transition between the leader and follower roles, and its timing, is necessary to translate progressive negotiation and hesitation. When switching abruptly between these states, the only way to translate hesitation is to oscillate from one state to the other while trying to decide what to do. On the contrary, if the switching is smooth, the role redistribution and sharing is progressive. This allows each partner to have knowledge and understanding on what the collaborative partners intents are.

Note that reasoning in the task space is interesting in regards to the control viewpoint and the existing algorithms, but may appear to be limited since different behaviors (namely interaction forces) can lead to exactly the same task. Now, we need to define clearly what leader and follower mean.

First, this notion of leader and follower is not common in the sensory-motor control community. Moreover, knowledge on dyad physical interaction is few compared to the amount of knowledge available for human free or specifically constrained motion (such as pointing or walking respectively). Understanding sensory-control mechanisms and models on how dyads perform in physical interaction tasks is beyond the scope of this paper. We are rather interested in catching a model from intuition that can be used to be programmed on a virtual avatar or on a humanoid robot collaborating with a human operator. Therefore, we assume that the simulated or machined avatars could be programmed by suitable controllers. One interesting characteristic of our approach is that it does not depend on the form of the controllers that are chosen to describe the two extreme behaviors for each single user. In fact, we are not sure whether humans use (i) two distinct controllers to switch from a leader to a totally passive follower or, (ii) a single controller with adaptable gains to switch from these extreme behavioral cases. This is a very challenging question and to our best knowledge, what we have in hands now is that human adjust impedance according to the tasks. Another issue, evidence that can be stated as a conjecture, is that whatever the controller form or nature is, the switching between the two behaviors is nicely continuous by the very nature of our metabolism and motor-sensory characteristics.

In the following, we introduce some mathematic basis followed by the model which we believe accounts for most of these observations.

\subsection{Homotopy}

Definition 2.1 A homotopy between two continuous function maps $f: X \rightarrow Y$ and $g: X \rightarrow Y$, where $X$ and $Y$ are topological spaces, is defined to be a continuous function map $h: X \times[0,1] \rightarrow Y$ such that:

$$
h(x, 0)=f(x) \text { and } h(x, 1)=g(x)
$$

Assuming the second parameter $h$ to be the time, then $h$ describes a "continuous deformation" of $f$ into $g$. At time 0 we have the function $f$ and at time 1 we have the function $g$.

Now, for a given operator (human, avatar, or robot) if we think of $f$ as the leader behavior controller and $g$ as the follower behavior controller, what remains is to define the homotopy $h$. Here the homotopy $h$ can be seen as the controller which allows a continuous switching function for each operator to balance between two behaviors. Intuitively, the homotopy allows modeling two distinct sensory-motor controllers and adaptively adjusting their weighting at will.

However, if the controller functions $f$ and $g$ have the same analytical form but have different parameters, then the reasoning would simply mean that we are dealing with a particular homotopy (interpolation) between the parameters. In other words, if a human uses a similar impedance control function when s/he is active or passive, but the gains of the impedance are adjusted for each behavior, the homotopy occurs simply at the gains' level.

\subsection{A POP homotopy bilateral coupling}

Now we will apply the previous theory to our POP case study. Our assumption, to be as much generic as possible, is that an operator 
has a specific leader-behavior's controller and a specific followerbehavior's controller. There is a large variety of possible expressions for $h$ and determining the right one is likely another challenging open investigation if it appears that the model is valid. We will borrow from control theory to describe the model. Let $u_{i}$ be the control signals for operator $i$. Let $h_{i}$ be the homotopy function for the operator $i$. We then propose the controllers $u_{i}$ as a linear homotopy $h(x, \alpha)$ having the following form:

$$
u_{i}=\alpha_{i} \mathscr{L}_{i}+\left(1-\alpha_{i}\right) \mathscr{F}_{i}
$$

$\alpha_{i}$ are functions of time and will evolve separately for each operator, depending on tasks constraints and on the will of each operator. Actually, this can be seen as a template model. Both $\mathscr{L}$ and $\mathscr{F}$ work as closed-loop filters on the difference between desired intention and actual motion. Basically, $\mathscr{L}$ and $\mathscr{F}$ can have the same analytical form or they can operate on different modalities and have different expressions, as in the example presented later in this paper. leader
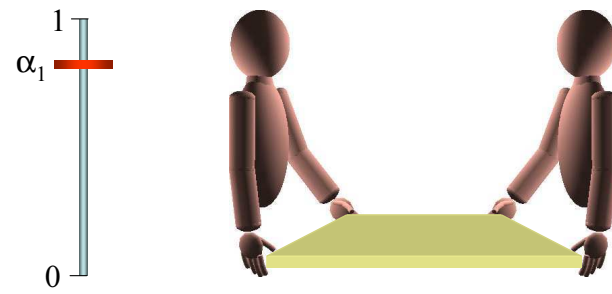

follower leader

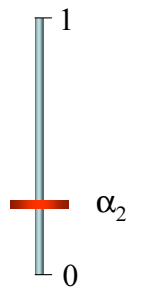

follower
Figure 1: Illustration of one degree of freedom homotopy for each individual of the physical interaction task dyad (holding a table). Each $\alpha_{i \in\{1,2\}}$ may evolve independently from the other and their time function results on a dynamic sliding between 0 and 1 during task execution.

Assuming we have two operators $i \in 1,2$, the task consists in porting an object from a place to another. We assume that both operators are identical and that they are behaving with limited cognitive knowledge. However, each operator has its own planned path motion for the object being manipulated. The Figure 1 illustrates the setup and also the planned (intended) trajectory for each operator. Intuitively, when the trajectories are the same, it is likely that no conflicting situation occurs; on the contrary, different desired trajectories induce a situation of conflict that need to be negotiated and resolved.

Let $P_{i}^{d}(t)$ be the desired trajectory for operator $i, P_{i}$ the actual trajectory for operator $i$, and $F_{i}(t)$ the interaction force sensed by operator $i$. Let us define $\varepsilon_{i}=P_{i}^{d}-P$ as the tracking error. Assuming that we choose the simple following expressions for the controllers:

$$
\begin{aligned}
& \mathscr{L}_{i}=J_{i}^{+} \lambda_{p} \varepsilon_{i} \\
& \mathscr{F}_{i}=-J_{i}^{+} \lambda_{f} F_{i}
\end{aligned}
$$

where $J^{+}$is the pseudo-inverse of the Jacobian, $\lambda_{p}, \lambda_{f}$ are given controller gains. Since both operators are connected through an object, we can write:

$$
\begin{aligned}
& u_{1}=\alpha_{1} J_{1}^{+} \lambda_{p} \varepsilon_{1}-\left(1-\alpha_{1}\right) J_{1}^{+} \lambda_{f} F_{1} \\
& u_{2}=\alpha_{1} J_{2}^{+} \lambda_{p} \varepsilon_{2}-\left(1-\alpha_{2}\right) J_{2}^{+} \lambda_{f} F_{2}
\end{aligned}
$$

Let us examine what happens with this bilateral coupling equations when both $\alpha_{i \in 1,2}$ vary.
- $\alpha_{1}=0$ and $\alpha_{2}=0$ correspond to the extreme situation where both operators are followers; in other words they both behaves passively in regards to the object. If the object is has zero mass, nothing happens and in the contrary, both operators will accompany its falling.

- $\alpha_{1}=1$ and $\alpha_{2}=1$ correspond to the extreme situation where both operators are masters, that is both behaves actively in regards to the object. Clearly, in time intervals where the both desired trajectories overlap, the trajectory is tracked, otherwise, the most powerful operator wins. Such a conflicting situation may lead to a blocking situation (when both operators have exactly the same power capabilities and each one sticks to his/er own intentions).

- $\alpha_{1}=1$ and $\alpha_{2}=0$ or $\alpha_{1}=0$ and $\alpha_{2}=1$ correspond to the extreme situation where one operator is the perfect master and the other the perfect follower. The follower will act passively regarding the forces applied by the leader that are transmitted through the manipulated object, and hence follow the leader. Clearly, in time intervals where the both desired trajectories overlap, the trajectory is tracked, otherwise, the master operator conduct the object according to his intended trajectory.

Now, these are extreme cases. In this paper, we make the hypothesis that the homotopy variables $\alpha_{i}$ will vary differently from each other and in a continuous way between 0 and 1 . We also assume that $\alpha_{i}$ are rather vectors $\vec{\alpha}_{i}$ of scalars having the dimension of the task space, and that the control behavior of each operator of the dyad is rather a weighting between the two extreme cases. This weighting may link to internal forces and the variation of a pair of component in $\vec{\alpha}_{i}$ describes, somehow, the physical interaction signature of the task.

Now that the model is established a question arises whether, for a given task and its constraints circumstances, the homotopy functions can be identified from real experiments? This model is certainly generic and hypothetical. Therefore even if valid, subjectivity of the person and the variability of the constraints and the environment contexts, in which a similar task can be performed, induces very likely a variability of the way both vectors $\alpha_{i}$ vary. In general, we believe that it is difficult to consider these vector functions as signature of tasks; however, they can be tuned to reflect specialization $[15,14]$ or conflicts, etc. This is an open issue that will be thoroughly investigated in future work.

\subsection{Genericity}

Our model is an abstract model and can be implemented in various ways, using very sophisticated controllers for $\mathscr{L}_{i}$ and $\mathscr{F}_{i}$. A potential implementation for the leader controller would be the one proposed in [2], where the follower robot can be a more or less active follower. Any level of sophistication can be chosen for the implementation, including implementation based on stack of tasks [17] or operational space formulation [7].

We can also implement our model as a high level controller that would give an input to a lower level impedance controller, following the idea of [6]:

$$
\begin{aligned}
& X_{0}^{i}=\alpha_{i} \mathscr{L}_{i}+\left(1-\alpha_{i}\right) \mathscr{F}_{i} \\
& F_{i}=M_{i} \ddot{V}_{i}+B_{i} \dot{V}_{i}+K_{i} V_{i} \\
& V_{i}=X_{o}^{i}-X_{i}
\end{aligned}
$$

According to the impedance control model, our controller would give an input trajectory to a low level impedance controller. The input trajectory would be defined by a homotopy between the trajectory that the partner would follow as a leader and the one s/he would follow as a follower. The dynamic behavior (i.e. how the partner reacts to disturbances while tracking own desired trajectories) would then be defined by the gains of the low level impedance controller. These gains can be set according to the context of task execution, on stability criteria, or even be a function of the homotopy variable, so that the final controller is a homotopy between two impedance controllers. This is richer than interpolating the gains between follower 


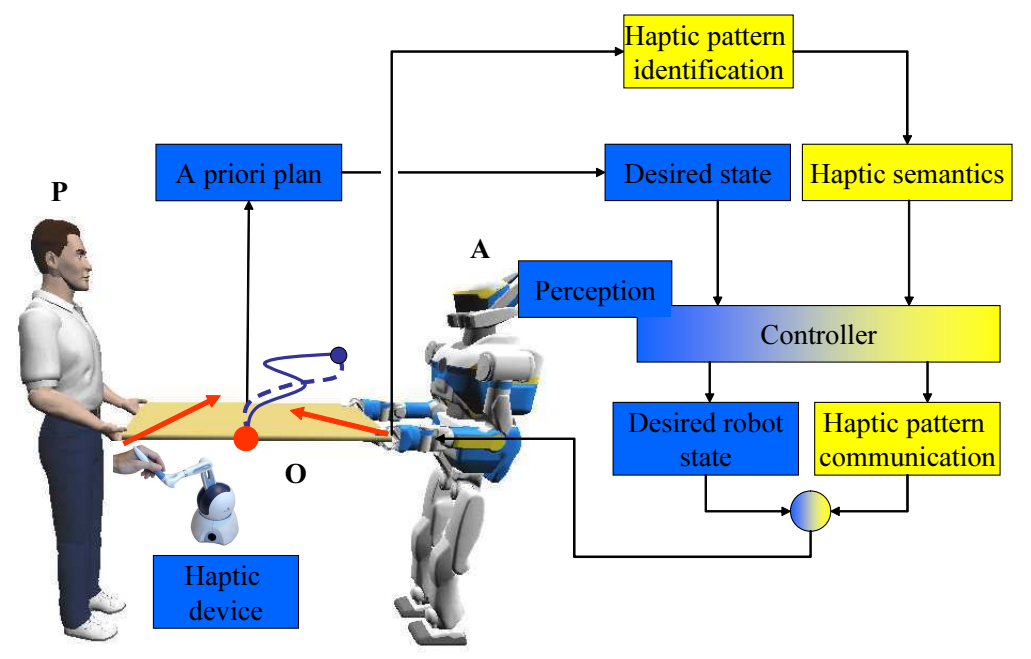

Figure 2: Person-Object-Avatar physical collaborative task architecture and main modules.

gains and leader gains, as the leader-mode impedance controller is not constrained to have fixed gains: depending on the context and the behavior of the follower, he can adapt his gain to optimize energy consumption, or adapt them to have a better disturbance rejection.

\section{Applications}

In dyad POP, when a human operator $(\mathrm{P})$ is replaced by a robotic or a virtual avatar (A), the POP scenario is transformed into POA. Our concern with this study is to realize physical collaborative POA tasks where the avatar is not affixed in an exclusive follower role, but may, as we humans do, be active and autonomous. In other words, we would like to enhance robotic or virtual avatars with physical interaction cognition so that they become more humanlike partners in physical collaborative tasks, see Figure 2.

Our newly proposed approach to physical interaction provides a model on which a behavioral architecture could be built on. These computer and machine avatars are indeed simpler than humans: they can be programmed with any controller model. The overall physical interaction architecture is made from the following components:

The avatars basic controllers consist in the algorithms for $\mathscr{L}$ and $\mathscr{F}$. Once a task is defined, we can provide the avatar with $a$ priori trajectories or with appropriate sequencing of elementary motions which realize the task. This is represented by the dashed curve linking the starting and ending points of the table on Figure 2. Note that each partner may have a different planed trajectory to realize the same task. This is illustrated by the continuous line linking the starting and ending points supposed to be the human desired trajectory.

The haptic pattern identification module is of prime importance in our approach. Since it is difficult to know a priori the vector function $\vec{\alpha}_{H}$, it need to be estimated on-line, during task execution. We believe that the haptic cue is composed of part of the signal which is relevant to the task achievement and that the remaining part can be seen as relevant to communication or synchronization. Haptic pattern identification is likely based on time-integral of internal forces or the time-derivative of forces; the last being problematic when force sensors (simulated or real) are used. This issue is a challenging open problem in haptic research.
The haptic semantic module ideally provides useful interpretation of the haptic patterns according to the task and the environment context. Ideally it would also merge information from other percepts. This module helps the controller block in selecting suitable rules or behaviors for a continuous adjustment for $\vec{\alpha}_{A}$ (yellow part), eventually combined with a desired state of the avatar (blue part).

The haptic patterns communication is simply a hypothetical module which generates distinct haptic patterns (signals) on top of or blended with the control signal of the avatar: its main mission is to try to communicate avatar intention to the human operator through the haptic channel.

Part of these components has been implemented in a heuristic way in this paper. Our preliminary goal is to show that various physical interaction behaviors can be implemented and simulation can be achieved. We used the AMELIF framework proposed in [4] that we enhanced with these modules. We implemented a collaborative task scenario in which the virtual avatar is the humanoid robot HRP-2 model and the user interacts with the simulation through a haptic device. This application has potential use in futuristic cognitive interactive games. We can imagine the possibilities that are offered by providing game-users/developers with such potential extensions.

\subsection{Experimental setup}

We simulated an object lifting task in which a human operator cooperates with a virtual humanoid robot. The human partner operates through a PHANToM Desktop haptic device and has a 3dimensional force feedback (see Figure 3). The goal of the task is to move an object from one point to another by passing over an obstacle. Figure 4 shows part of the virtual scene and the trajectory of the object desired by robot to complete the task. This trajectory is unknown to the human partner and is not displayed during the task. We conducted experiments in an informal way, on one subject, as the goal of the paper is to introduce ideas and evaluate their feasibility rather than exposing final results.

\subsection{Control}

As the leader and follower controllers, we used the laws presented in subsection 2.3. In a first set of experiments, $\alpha$ was varied in a time-dependent way, so that the robot was leading at some points in the task. The task was either not divided, or divided into two or 


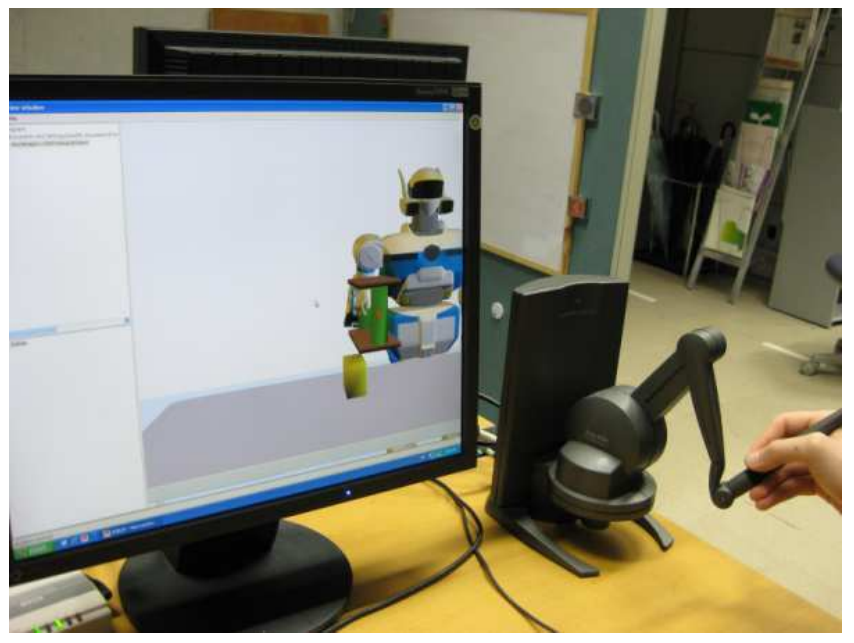

Figure 3: Experimental setup.

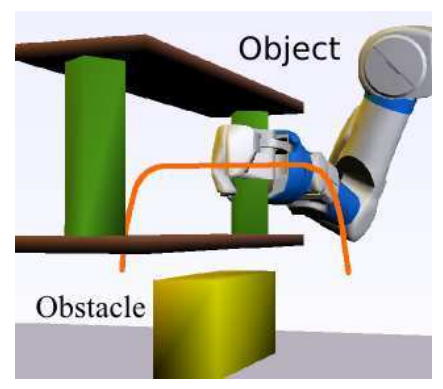

Figure 4: Plan of the virtual avatar: desired motion of the object.

three parts, depending on the time-profile of the homotopy variable. We used the following time profiles:

- $L$ : the virtual avatar leads all along the task.

- $F$ : the virtual avatar follows the human all along the task.

- $L-F(\mathrm{r} F-L)$ : the virtual avatar leads (follows) during the first half of the motion, and follows (leads) the human at the end of the motion.

- $L-F-L(F-L-F)$ : the avatar leads (follows) during the lifting and landing phases only, while the human operator leads (follows) while the object passes over the obstacle.

\subsection{Preliminary results and discussion}

One important thing to consider when switching between two controllers in such a linear way is stability. Though we did not tackle this issue theoretically, we plotted the output of the controller along the task to check its smoothness during the transitions between the leader's and follower's states. Figure 5 shows a typical joint torque output from the homotopy controller. The signal appeared to be smooth, with higher torques during the transitions.

We could not highlight specialization from the force applied by the subject and his virtual partner on the object. This result was expected from [13]. When the human user talked about his impressions on how the robot virtual avatar behaved, he explained that he did not trust the avatar, and thus applied a "safety" force, even when he felt like the avatar was leading the task, to be sure to avoid the obstacle. This is a probable reason why we could not observe specialization. Moreover, we only looked for the same functional specialization as the one discovered by Reed, which consist in specializing in the acceleration and deceleration of the object. As our task is more complex, other specializations might have been elected
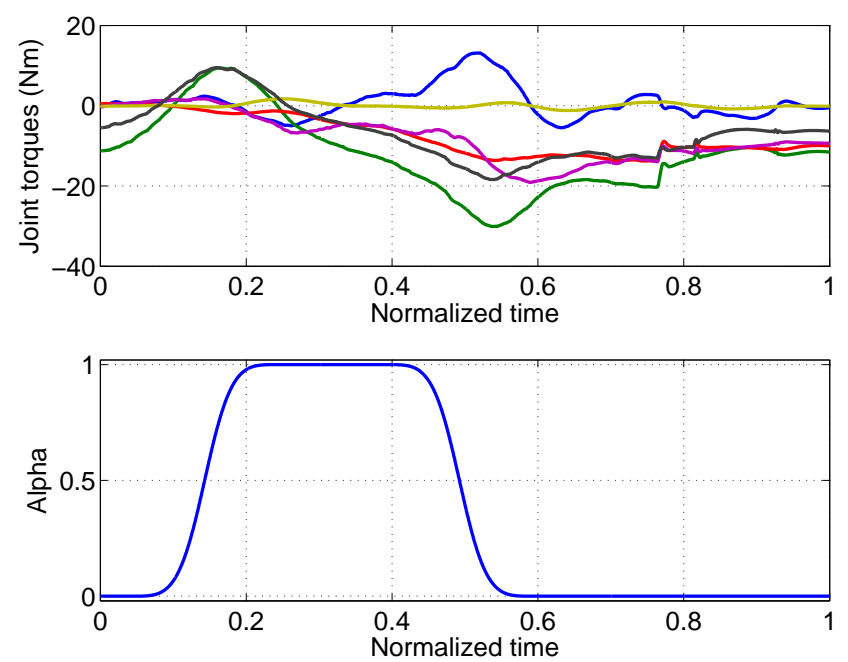

Figure 5: Typical joint torques at the joints of the avatar (chest joint and right arm joints). The torque references sent to the actuators of the robot are smooth even when the value of the homotopy variable changes.

by human partners performing the task.

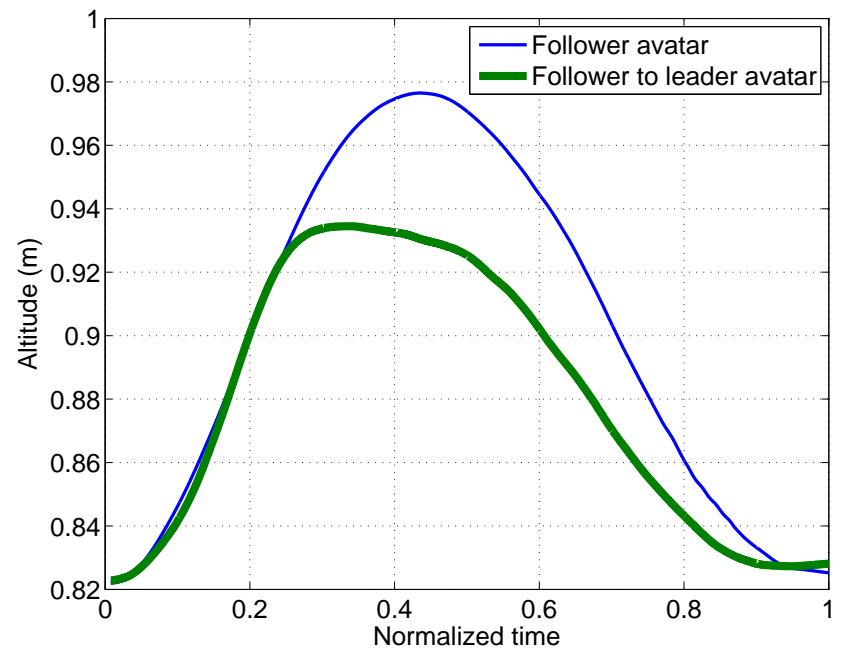

Figure 6: Trajectories when the human leads all the task (blue) and when he leads part of the task (green). The human trajectory is smoother than the one of the avatar and goes higher (further from the obstacle).

Questioned about his experience, the subject reported that he felt more comfortable when the avatar was following him during the lifting and landing phase ( $F-L-F$ time profile of the homotopy variable $\alpha$ ), and especially when being close to the corners of the object, as he did not trust the virtual avatar. He also reported that the robot was suggesting him a more time-optimal trajectory, as it was moving much closer to the object than the subject would have done. Figure 6 highlights this fact by showing a typical trajectory of the object when the subject lead the task and when the robot was leading part of it. Note that when the robot is a follower all along the task, the trajectories of the object are very close to the ones reported in [10], while the trajectory desired by the avatar is more square-shaped as it closely follows the contour of the object 
(thus leading to more jerky motions). Hence, the reference path of the avatar is maybe unnatural to the human operator, which could explain why he did not follow the avatar passively.

Surprisingly, though the subject was presented several trials where the avatar was leading the entire task, the subject felt like it was never the case. This might be related to the stiffness of the leader controller $\mathscr{L}$. Maybe the controller did not offer enough disturbance rejection, thus giving the subject the feeling that he had some control over the task.

Finally, it appeared that the subject was somehow disrupted at first when the robot changed his homotopy variable during the task. It took time for him to understand what was happening. A similar result has been reported in [2], in which subjects felt that a robot varying his level of assistance during the task, rather than between tasks, was unnatural. Our time profiles of the homotopy variable corresponded to artificial specialization, which was applied without taking into account contextual aspects or force signals, and which started abruptly after a set of trials in which the robot was a pure follower. This scenario seems not very probable in real-life situations, as Reed reported that specialization emerged quickly, but after several trials [16]. In future work, we plan to investigate on how to define the homotopy variable depending on the task, its context and the forces perceived by the virtual avatar so that its behavior is more user-friendly. This might require to use a more appropriate implementation of our abstract model (in other words, to change the $\mathscr{L}$ and $\mathscr{F}$ controllers)

\section{Conclusion}

The motivation of our work is to establish a physical haptic interaction model which does not appoint any agent composing a dyad to be in either a follower or leader role. Our approach consists in considering that in realizing a given task, each individual would behave in an extreme case as either an agent who imposes his intention suspecting from the collaborator to be a gentle follower or in a reverse way. We believe that in reality these extreme cases are rarely reached and made the hypothesis that each individual behave in a continuous weighted control between these two extreme case. This tuning is realized by a homotopy (interpolation) switching between either two distinct controllers (one ensuring a follower behavior the other one the leader behavior), or between two sets of gains for a single controller (case of adjustable impedance). We showed that the homotopy describes a large number of physical interaction behaviors, adjustment of which can be linked to combined contextual and haptic communication cues. We implemented this idea thanks to the simplicity of the model that can be considered as a template and made preliminary trials that raise several issues that we aim to address as future work:

- if this model is valid in POP scenarios, how can we identify the homotopy functions and variables?

- how can the homotopy variable be adjusted according to the contextual and haptic communication cue to realize automatic specialization and natural collaboration?

- how tasks can be decomposed in appropriate subtasks and/or complementary sub-spaces?

- does the homotopy variable correlate to internal forces?

A more technical question remains to guarantee unconditional stability of the homotopy.

\section{ACKNOWLEDGEMENTS}

This work is partially supported by grants from the ImmerSence EU CEC project, Contract No. 27141 www.IMMERSENCE.INFO (FET-Presence) under FP6.

\section{REFERENCES}

[1] H. Arai, T. Takubo, Y. Hayashibara, and K. Tanie. Human-robot cooperative manipulation using a virtual nonholonomic constraint. In IEEE Internationl Conference on Robotics and Automation, pages 4064 4070, San Francisco, CA, April 2000.

[2] B. Corteville, E. Aertbelien, H. Bruyninckx, J. D. Schutter, and H. V. Brussel. Human-inspired robot assistant for fast point-to-point movements. In ICRA, pages 3639-3644. IEEE, 2007.

[3] V. Duchaine and C. M. Gosselin. General model of human-robot cooperation using a novel velocity based variable impedance control. In WorldHaptics, pages 446-451, Washington, DC, USA, 2007.

[4] P. Evrard, F. Keith, J.-R. Chardonnet, and A. Kheddar. Framework for haptic interaction with virtual avatars. In 17th IEEE International Symposium on Robot and Human Interactive Communication (IEEE RO-MAN 2008), Munich, Germany, August 2008.

[5] Y. Hirata and K. Kosuge. Distributed robot helpers handling a single object in cooperation with a human. In IEEE Internationl Conference on Robotics and Automation, pages 458-463, 2000.

[6] N. Hogan. Impedance control: an approach to manipulation, Part 1. Journal of Dynamic Systems, Measurement, and Control, 107:17, March 1985.

[7] O. Khatib. A unified approach for motion and force control of robot manipulators: The operational space formulation. Robotics and Automation, IEEE Journal of [legacy, pre - 1988], 3(1):43-53, 1987.

[8] K. Kosuge, H. Yoshida, and T. Fukuda. Dynamic control for robothuman collaboration. In Proceedings of the 2nd IEEE International Workshop on Robot and Human Communication, pages 389-401, Tokyo, Japan, 1993.

[9] Y. Maeda, T. Hara, and T. Arai. Human-robot cooperative manipulation with motion estimation. In IEEE/RSJ International Conference on Robots and Intelligent Systems, pages 2240-2245, Maui, Hawaii, October-November 2001.

[10] S. Miossec and A. Kheddar. Human hand motion in cooperative tasks: moving object case study. In IEEE International Conference on Robotics and Biomimetics, page (to appear), Bangkok, Thailand, December 14-17 2008.

[11] A. Peer, S. Hirche, C. Weber, I. Krause, M. Buss, S. Miossec, P. Evrard, O. Stasse, E.-S. Neo, A. Kheddar, and K. Yokoi. Intercontinental multimodal tele-cooperation using a humanoid robot. In IEEE/RSJ International Conference on Intelligent RObots and Systems, pages 405-411, Nice, France, 2008.

[12] M. Rahman, R. Ikeura, and K. Mizutani. Investigation of the impedance characteristic of human arm for development of robots to cooperate with humans. JSME international journal. Series $C, M e$ chanical systems, machine elements and manufacturing, 45(2):510518, 2002.

[13] K. Reed, J. Patton, and M. Peshkin. Replicating human-human physical interaction. In Proceedings of the 2007 IEEE International Conference on Robotics and Automation (ICRA 2007), 2007.

[14] K. Reed, M. Peshkin, M. Hartmann, J. Patton, P. Vishton, and M. Grabowecky. Haptic cooperation between people, and between people and machines. In 2006 IEEE/RSJ International Conference on Intelligent Robots and Systems, pages 2109-2114, 2006.

[15] K. B. Reed, M. Peshkin, M. J. Hartmann, J. E. Colgate, and J. Patton. Kinesthetic interaction. In In International Conference on Rehabilitation Robotics (ICORR), pages 569-574. IEEE, 2005.

[16] K. B. Reed and M. A. Peshkin. Physical collaboration of humanhuman and human-robot teams. IEEE Transactions on Haptics, 1(2):(to appear), August-December 2008.

[17] O. Stasse, A. Escande, N. Mansard, S. Miossec, P. Evrard, and A. Kheddar. Real-time (self)-collision avoidance task on a hrp-2 humanoid robot. In IEEE International Conference on Robotics and Automation,ICRA-08, Pasadena, California, on May 19-23, 2008, pages 3200-3205, 2008.

[18] T. Tsumugiwa, R. Yokogawa, and K. Hara. Variable impedance control with regard to working process for man-machine cooperationwork system. In IEEE/RSJ International Conference on Robots and Intelligent Systems, pages 1564-1569, Maui, Hawaii, OctoberNovember 2001. 